\title{
Special volume, preface - 9th International Workshop on Astronomy and Relativistic Astrophysics: From Quarks to Cosmos
}

\author{
César A. Zen Vasconcellos ${ }^{1,2} \mid$ Peter O. Hess $^{3,4} \mid$ Gabriella Piccinelli $^{5} \mid$ Mariana Vargas $^{2}$ \\ Magaña $^{6} \mid$ Luis Ureña-Lopez $^{7}$ | Ricardo Gonzalez Felipe ${ }^{8,9} \mid$ Thomas Boller $^{10} \mid$ Steven \\ Gullberg $^{11}$
}

${ }^{1}$ Universidade Federal do Rio Grande do Sul - UFRGS, Porto Alegre, Brazil

${ }^{2}$ International Center for Relativistic Astrophysics Network - ICRANet, Pescara, Italy

${ }^{3}$ Universidad Nacional Autónoma de México - UNAM, México City, Mexico

${ }^{4}$ Frankfurt Institute for Advanced Studies, J.W. von Goethe University, Hessen, Germany

${ }^{5}$ Centro Tecnológico, Facultad de Estudios Superiores Aragón, Universidad Nacional Autónoma de México, Nezahualcóyolt, Mexico

${ }^{6}$ Instituto de Física, Universidad Nacional Autónoma de México, Ciudad de México, Mexico

${ }^{7}$ Departamento de Física, Universidad de Guanajuato, León, Mexico

${ }^{8}$ Instituto Superior de Engenharia de Lisbon - ISEL, Lisbon, Portugal

${ }^{9}$ Centro de Física Teórica de Partículas CFTP/IST, Lisbon, Portugal

${ }^{10}$ Max-Planck Institut für

Extraterrestrische Physik - MPI, Garching bei München, Germany

${ }^{11}$ University of Oklahoma - OU and International Astronomical Union - IAU, Norman, Oklahoma

\section{Correspondence}

César A. Zen Vasconcellos, Universidade Federal do Rio Grande do Sul - UFRGS and International Center for Relativistic Astrophysics Network - ICRANet,

Pescara, Italy.

Email: cesarzen@cesarzen.com

\begin{abstract}
This special volume includes contributions from some of the most relevant scientists who have participated in the IWARA 2020 Video Conference. The series of meetings known by the acronyms IWARA, STARS, and SMFNS,--more than a congregation of events, students and researchers which in itself would be extremely important for science-became in last years a kind of platform for scientific and academic projects, partnerships and presentation of high-quality research contributions describing original and unpublished results on several topics related as new phenomena and new states of matter in the Universe, general relativity, gravitation, cosmology, astrophysics, particle and nuclear physics, and topics related. And the scientists participating in this volume, perfectly integrated with this design, bring more than just a glimpse into the technical toolbox of their work, be it theoretical or experimental. In addition to international scientific recognition, these researchers are mentors of students and young researchers, and demonstrate, in these contributions, also glimpses of systematically complex decisions made about the interpretation of data, about which problems are relevant to pursue, thus contributing a lot to the craft of science and its development.
\end{abstract}

\section{K E Y W O R D S}

cosmos, IWARA, quarks, SMFNS, STARS 


\section{1 | THE IWARA 2020 VIDEO CONFERENCE}

The 9th International Workshop on Astronomy and Relativistic Astrophysics-IWARA2020 Video Conference was held from September 6-12, 2020, in virtual format. IWARA 2020 was the 20th edition of a set of analytically continued events known by the acronyms IWARA, STARS, ${ }^{1}$ and SMFNS, ${ }^{2}$ and required an extraordinary effort by the organizers in order to rearrange an event that was originally scheduled to be in person.

In this preface, while not describing the experience of organizing an event with such characteristics (for a detailed information about the event and the details of its organization see [Vasconcellos et al. 2021]), we wish to reaffirm what we stressed before: that despite the pandemic has profoundly touched the lives of all human beings across the planet, affecting mobility, the relationship between persons and their surroundings, and even our perception of time and space, it has created a feeling of extreme empathy with the suffering of many human beings around the world combined with another type of feeling, that of being stuck in our present, without being able to plan daily activities in advance. This pandemic greatly affected this way the organization of the meeting, whose main activities were scheduled in advance by a group of selfless people located at the meeting place and distant collaborators. A change of course was necessary.

We also would like to reaffirm as stressed before, that the results of the IWARA 2020 Video Conference are remarkable. The event had 35 invited live lectures, 52 lectures from the parallel sessions, and 66 poster presentations, with most of the authors of the parallel and poster sessions participating in the live spotlight plenary sessions. In addition, we have a remarkable number of videos stored on the YouTube channel (128), with 4,834 views and 189 subscribers on this channel. An important aspect to be noted is that we had 384 subscribers, from 41 countries, as shown in the map in Figure 1.

It remains to thank once more the members of the Local Organizing Committee, the International Organizing Committee, and the International Advisory Committee (for the complete list of names please see [Hess \& Vasconcellos 2020]) as well as the technical staff of the University of Oklahoma who provided the webinar operating system for the meeting. It is also important to thank the Astronomische Nachrichten (AN), in the person of its Editor Klaus G. Strassmeier, as the AN has been a partner of unparalleled importance in the publication of the proceedings of the series of events known by the acronyms IWARA, STARS, and SMFNS. With regard particularly to the IWARA 2020 Video Conference event, AN accepted

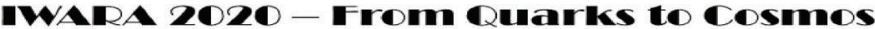

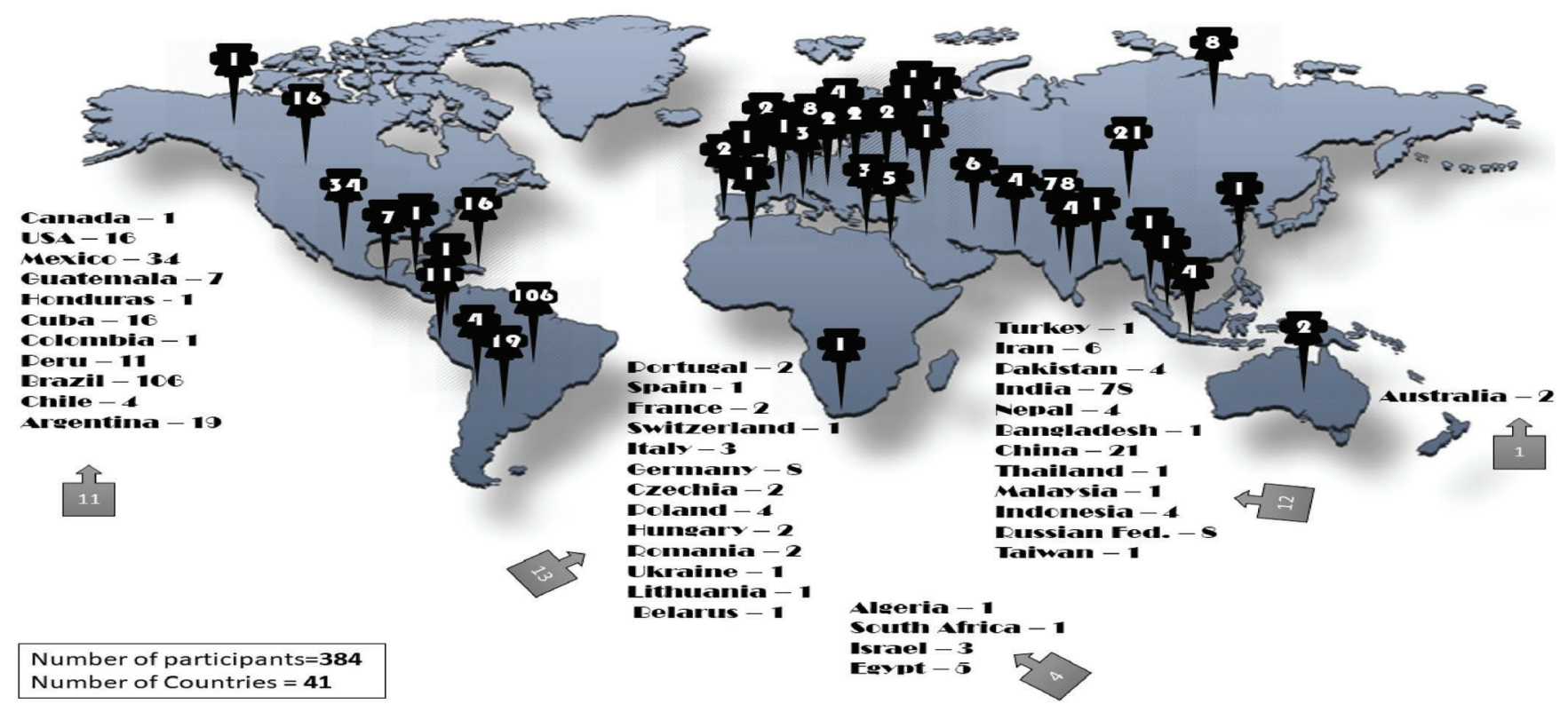

F I G U R E 1 World map of the participants in the IWARA 2020 Video Conference

${ }^{1}$ Acronyms for Caribbean Symposium on Cosmology, Gravitation,

Nuclear and Astroparticle Physics.

${ }^{2}$ Acronyms for International Symposium on Strong Electromagnetic

Fields and Neutron Stars. 
gladly to publish, in addition to the proceedings of the meeting, this special volume with keynote speakers.

For more information about virtual events, we recommend reading: (Frisch \& Greene 2020; Hugel 2020; Kassab 2020; Virtway 2019; Writer 2020). For more information about the IWARA2020 Video-Conference, please visit: (Hess \& Vasconcellos 2020; Vasconcellos et al. 2020; Vasconcellos et al. 2020a, 2020b, 2020c, 2020d).

Our expectations now turn on to the IWARA 2022 meeting, that we hope to be held in person (with some elements of virtuality), in Guatemala, in September 2022, at a date and place to be specified by the local committee. For those interested, the chair of the event will be Dr José Rodrigo Sacahui Reyes, from the University of San Carlos de Guatemala, email: jrsacahui@gmail.com. Until then.

\section{REFERENCES}

Frisch, B., \& Greene, C. 2020, What It Takes to Run a Great Virtual Meeting. Available at https://hbr.org/2020/03/what-it-takes-torun-a-great-virtual-meeting

Hess, P. O., \& Vasconcellos, C. A. Z. 2020, IWARA Committiees. Available at https://indico.cern.ch/event/822124/page/ 17110-committees

Hugel, M. 2020, Virtual Events Vs. In-Person Events: Why You Should Host Your Event Online. Available at https://blog. workcast.com/virtual-events-vs-in-person-events

Kassab, K. 2020, Technically speaking: What's Behind a Virtual Event. Available at https://www.hartmannstudios.com/ blog/virtual-event-tech

Vasconcellos, C. A. Z., Hess, P. O., Piccinelli, G., et al. 2020, Special Volume, Preface - 9th International Workshop on Astronomy and Relativistic Astrophysics: from Quarks to Cosmos. Available at https://onlinelibrary.wiley.com/doi/10.1002/asna.202113874

Vasconcellos, C. A. Z., Hess, P. O., Magaña, M. V., \& Bodmann, B. 2020a, IWARA - From Quarks to Cosmos. Available at https:// www.youtube.com/channel/UCdU8tqakcpnohGUI0CEanYw? view_as=subscriber
Vasconcellos, C. A. Z., Hess, P. O., Magaña, M. V., \& Bodmann, B. 2020b, IWARA - From Quarks to Cosmos. Available at http:// www.cesarzen.com/IWARAplatform/index.html

Vasconcellos, C. A. Z., Hess, P. O., Magaña, M. V., \& Bodmann, B. 2020c, IWARA - From Quarks to Cosmos. Available at https:// www.instagram.com/iwara2020

Vasconcellos, C. A. Z., Hess, P. O., Magaña, M. V., \& Bodmann, B. 2020d, IWARA - From Quarks to Cosmos. Available at https:// twitter.com/iwara2020

Vasconcellos, C. Z., Hess, P., Piccinelli, G., et al. 2021, Astron. Nachr., $342,18$.

Virtway. 2019, Face-to-face vs. Virtual Events vs. Hybrid. Available at https://www.virtwayevents.com/face-to-face-virtual-hybridevents

Writer, R. C. 2020, Virtual Events: A Step-By-Step Guide on How to Host a Successful Live Session. Available at https://en. rockcontent.com/blog/virtual-events/

\section{AUTHOR BIOGRAPHY}

Prof. César Zen Vasconcellos is a retired full professor of the Universidade Federal do Rio Grande do Sul (UFRGS), Porto Alegre, Brazil and a Adjoint Professor in the International Center for Relativistic Astrophysics Network (ICRANet), Pescara, Italy. His interests in physics cover a wide range of topics, specially theoretical nuclear physics, astroparticle physics, relativistic nuclear astrophysics, compact stars and cosmology.

How to cite this article: Zen Vasconcellos, C. A., Hess, P. O., Piccinelli, G., et al. 2021, Astron. Nachr., 342, 705. https://doi.org/10.1002/asna.202113986 